\title{
New All-Solid-State KTA-Based DIAL for Tropospheric Methane Monitoring
}

\author{
Taieb Gasmi Cherifi \\ Division of Science and Engineering, Saint Louis University-Madrid campus, \\ Avenida del Valle, 34. Madrid 2803, Spain
}

\begin{abstract}
We present an Optical Parametric Oscillator (OPO)-based differential absorption lidar (DIAL) that operates in the mid-infrared (3.0-4.5 um). Tunability of the OPO is achieved by the use of Potassium Titanyle Arsenate $\left(\mathrm{KTiOAsO}_{4}\right)$, or KTA crystal. The Dial measurements of methane are in good agreement with reported values and also exhibits diurnal fluctuations in concentration levels during atmospheric monitoring.
\end{abstract}

\section{INTRODUCTION}

We present a KTA crystal based-optical parametric oscillator pumped by a Q-switched $\mathrm{Nd}$ :YAG as a transmitter of a differential absorption lidar system. We will also conduct measurements of methane in an urban environment. The transmitter is a mid-IR laser source in a configuration based on a modified version of $\mathrm{V}$. Vaicikauskas ${ }^{1,2}$. The singly resonant $\mathrm{OPO}$, formed by two mirrors, contains a KTA crystal by Lasertec. In order to maximize the output energy, the cavity is carefully designed and its length is optimized to be $34 \mathrm{~mm}$. The output laser pulse has the maximum energy of $6 \mathrm{~mJ}$, pulse duration of $6 \mathrm{~ns}$ at a pulse repetition rate of 5-20 Hz. The KTA crystal of size $5 \times 5 \times 25 \mathrm{~mm}^{3}$ cut along $\mathrm{x}$-axis $\left(\theta=90^{\circ}, \varphi=0^{\circ}\right)$ satisfies the type II non-critical phase matching NCPM condition for optical parametric conversion. The crystal is not cooled and is wrapped with the indium foil and mounted on a copper block. The crystal is coated on both faces to have high transmission at 1064 $\mathrm{nm}, 1.5-1.6 \mu \mathrm{m}$, and $3.0-4.5 \mu \mathrm{m}$. The area monitored by the Lidar is a centric urban area where the main source of pollution can only be attributed to vehicle exhaust and to a very low extent to wind transport of methane from the further south industrial region.

\section{METHODOLOGY, RESULTS \& DISCUSSION}

Figure 1 illustrates the backscattered signal intensity as a function of the Lidar wavelengths which span the fundamental methane absorptions under investigation.

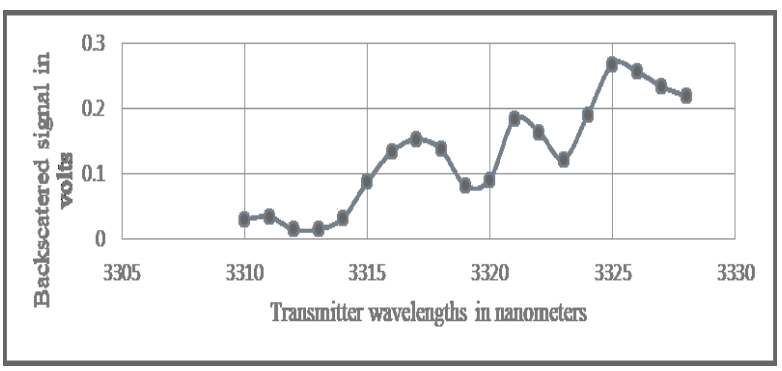

Fig.1: Backscattered signal intensity as a function of

the Lidar wavelength for methane monitoring

The mid-infrared spectrum of methane is characterized by a strong vibration-rotational band ${ }^{3,4}$, which is the Q branch $v_{3}\left(3016 \mathrm{~cm}^{-1}\right)$, which is located within the tunable range of OPO laser $(3-4 \mu \mathrm{m})$. The measurement of the urban concentration of the methane with high accuracy and sensitivity requires the prior knowledge of the transmission spectra of the atmosphere for a number of wavelengths from 3.0 to $3.45 \mu \mathrm{m}$. We have therefore made use of HITRAN2012 database $^{5}$ to identify the possible absorbing molecules in this band. This HITRAN edition has indeed witnessed major improvements within some hot bands for methane compared to HITRAN2008. It is worth mentioning that the mid-IR spectrum window contains absorption peaks for an extensive variety of trace gases ${ }^{6}$, including $\mathrm{H}_{2} \mathrm{O}, \mathrm{CO}, \mathrm{CO}_{2}, \mathrm{NO}, \mathrm{N}_{2} \mathrm{O}, \mathrm{NO}_{2}$ and $\mathrm{CH}_{4}$ which has a strong fundamental absorption at $3.3 \mu \mathrm{m}$. Moreover, the mid-infrared region 3.0$3.7 \mu \mathrm{m}$ is predominantly significant because it contains fundamentally all the characteristic 
stretching wavelengths of the molecules containing $\mathrm{C}-\mathrm{H}$ bonds. Water vapor does not have significant absorption in the region $3.15-$ $3.20 \mu \mathrm{m}$. Figure 2 shows the line intensity versus wavelength, generated based on the HITRAN, reveals clearly that the methane had strong absorption between $3.310 \mu \mathrm{m}$ and $3.317 \mu \mathrm{m}$. Based on these studies, we have selected 3.316 $\mu \mathrm{m}$ as online wavelength $\left(\lambda_{\text {on }}\right)$ and $3.326 \mu \mathrm{m}$ as an offline wavelength $\left(\lambda_{\text {off }}\right)$ to monitor atmospheric methane using our system with absorption $1^{7,8}$ cross section $\sigma_{\text {on }} \sim 3.497 \times 10^{-19} \mathrm{~cm}^{2}$ and $\sigma_{\text {off }} \sim 2.985 \times 10^{-}$ ${ }^{21} \mathrm{~cm}^{2}$.

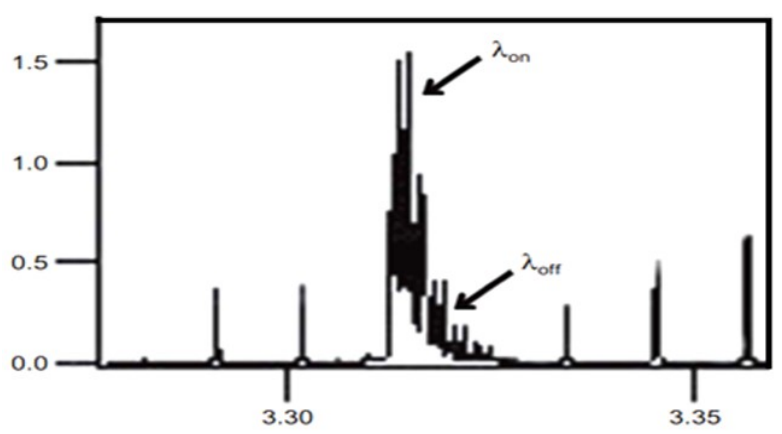

Fig. 2: Methane absorption spectra. Two selected lines $-\lambda_{\text {on }}$ and $\lambda_{\text {off }}-$ in the figure (source: HITRAN2012).

Figure 3 shows the experimental results of the variation of atmospheric methane as a function of time of the day. As it is well established that inversion layer is responsible for producing smog, trapping the pollutants produced by vehicles, fires and industrial activities. In our case, this effect is caused by the city topography that creates a temperature inversion since it can sometimes cause cold air to flow from the mountain peaks down into valleys where the city is located. The plateau concentration approached an average value of $1.8 \mathrm{ppm}$ of methane which is slightly higher than that of background (1.75 ppm). This decrease in methane concentration is provoked by the decrease in vehicle traffic coinciding with the increase in the atmospheric mixing depth in the afternoon. However, the tendency of methane concentration reverses during the late hours of the day at around 17:00 which could be attributed to second traffic rush hour of the day. It is therefore important to note the existence of diurnal fluctuations in concentration levels during atmospheric monitoring of $\mathrm{CH}_{4}$. This behavior is hence an additional evidence that urban areas are increasingly recognized as a globally important source of methane to the atmosphere; although, the location of urban methane sources and relative contributions of source sectors are not well known.

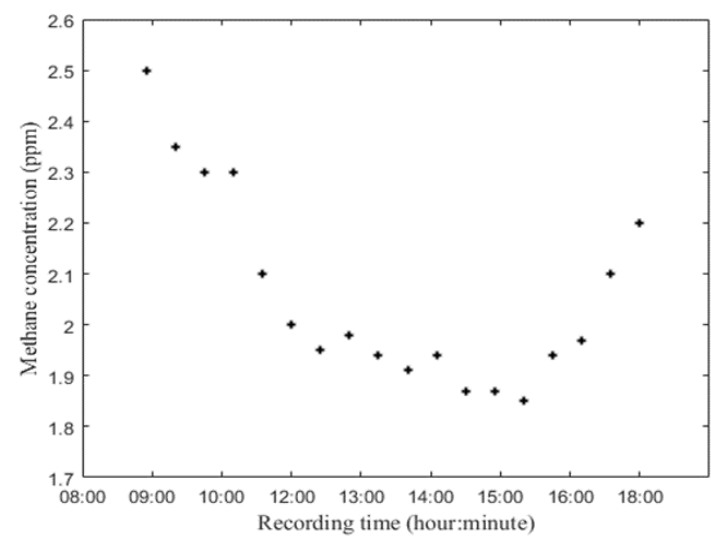

Fig. 3: Experimental results of the diurnal variation of atmospheric methane.

\section{REFERENCES}

[1] V. Vaicikauskasa, Z. Kuprionisb, M. Kaucikasa, V.Svedasa, V. Kabelkaa, "Mid-Infrared All-solid State DIAL for Remote Sensing of Hazardous Chemical Agents", Laser Radar Technology and Applications XI, Proc. of SPIE, 6214, (2006).

[2] Q.B.Sun, H.J. Liu, N. Huang, C. Ruan, S.L. Zhu and W. Zhao "High energy and High efficiency 3.4 um extracavity KTA optical parametric oscillator", Laser Phys. Lett. 8, No 1, 16-20 (2011).

[3] S.W. Lee, J. McNeil, T. Zenker, T.H. Chyba, "Methane Concentration Measurements with a Midinfrared Optical Parametric Oscillator-based Differential Absorption Lidar System", Proc. Lasers and Electro-Optics, CLEO/Pacific Rim '99. Seoul, SKorea, (1999).

[4] S.W. Lee, J. McNeil, T. Zenker, T.H. Chyba, "A Mid-IR OPO Based Lidar System for DIAL Measurements of Tropospheric Methane Concentration", Proc. Conference on Lasers and Electro-Optics, CLEO '99, Baltimore, MD, USA, (1999). 
[5] "The HITRAN2012 Molecular Spectroscopic Database", Journal of Quantitative Spectroscopy \& Radiative Transfer, 130, 130, 4-50, (2013).

[6] "Silicon on silicon dioxide slot waveguide evanescent field gas absorption sensor", M.A. Butt, S:N: Khonina and N.L. Kazanskiy, Journal of Modern Optics, Vol. 65, No 2, 174-178, (2018).

[7] S. Veerabuthiran, A. K. Razdan, M. K. Jindal, R. K. Sharma, V. Sagar. "Development of 3.0-3.45 um OPO Laser Based Range Resolved and Hard-target Differential Absorption Lidar for Sensing of Atmospheric Methane". Optics \& Laser Technology, 73, 1-5, (2015).

[8] R. J. Hargreaves, P. F. Bernath, J. Bailey, and M. Dulick, "Emperical Line Lists and Absorption Cross Sections for Methane at High Temperatures". The Astrophysical Journal, 813, 12, (2015). 